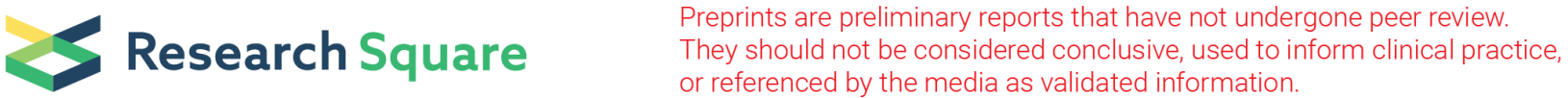

\section{Radiotherapy for Postsurgical Vaginal Recurrences of Cervical Squamous Cell Carcinoma: Analysis of Dosing and Prognosis}

\author{
Junfang Yan \\ Peking Union Medical College Hospital \\ Ziye Zheng \\ Peking Union Medical College Hospital \\ $\mathrm{Ke} \mathrm{Hu}$ \\ Peking Union Medical College Hospital \\ Xiaorong Hou \\ Peking Union Medical College Hospital \\ Lihua Yu \\ Peking Union Medical College Hospital \\ Fuquan Zhang ( $\square$ zhangfuquan3@126.com ) \\ Peking Union Medical College Hospital
}

Research Article

Keywords: radiotherapy, women's cancer, cancer management, prognostic factor

Posted Date: January 10th, 2022

DOI: https://doi.org/10.21203/rs.3.rs-1232060/v1

License: (c) (i) This work is licensed under a Creative Commons Attribution 4.0 International License. Read Full License 


\section{Abstract}

Background: This study aimed to investigate the efficacy of salvage radiotherapy for vaginal recurrence of cervical squamous carcinoma in patients who previously underwent surgery and to explore prognostic factors (particularly dose-related) associated with survival postrecurrence.

Methods: Ninety-seven patients with histologically proven squamous cell carcinoma-subtype cervical cancer who were treated for vaginal recurrence at Peking Union Medical College Hospital between July 2011 and November 2019 were identified. All patients had previously undergone surgery for the primary tumor and received salvage external beam radiotherapy, brachytherapy, or both. Factors predictive of overall survival (OS), progression-free survival (PFS), and local control (LC) were investigated, as were adverse effects.

Results: The median follow-up time was 42.5 months. The estimated 5-year OS, PFS, and LC rates were $84 \%, 79 \%$, and $91 \%$, respectively. On multivariate analysis, a tumor size $\leq 4 \mathrm{~cm}$ and an endovaginal recurrence pattern were associated with longer PFS (both $P<0.05$ ); however, only the latter was predictive of a longer LC $(P<0.05)$. In the 33 patients with recurrences that were paravaginal or invasive of surrounding organs, biologically equivalent doses in 2 Gy fractions of $\geq 70$ Gy were independently predictive of longer LC $(P<0.05)$. Finally, $12.4 \%$ of the patients experienced grades $\geq 2$ late complications; only 1 patient who received EBRT alone experienced grade 5 late complications.

Conclusions: RT is an effective treatment for post-surgical vaginal recurrence in patients with cervical squamous cell carcinoma. For patients with extravaginal recurrence, a salvage dose of $\geq 70$ Gy appears to be optimal.

\section{Background}

Cervical cancer is the fourth most common cancer among women [1] and the second most commonly diagnosed malignancy in developing countries [2]. Squamous cell carcinoma (SCC) is the most common pathological type of cervical cancer, accounting for approximately $80-90 \%$ of cases [3]; its prognosis differs from those of other pathological types [4-7].

Radical hysterectomy with pelvic lymphadenectomy is the standard recommendation for patients with early-stage cervical cancer [8]; however, the pelvic recurrence rate is $10-20 \%$ in patients with this disease after primary treatment, and the disease-free survival rate remains poor at $45 \%$ [9]. The vagina is the most common site of cervical cancer recurrence [10]. Treatment for recurrent cervical cancer that is confined to the upper vagina can be curative [11] yet remains challenging given the lack of a consistent standard treatment after the primary intervention. Pelvic exenteration is one of the main surgical methods for local recurrence; however, the perioperative mortality rate is relatively high, and the procedure may also reduce the patients' quality of life [12]. Salvage radiotherapy (RT) with or without concurrent chemotherapy is currently recommended owing to its effectiveness and tolerable adverse events [13-15]. External beam RT (EBRT) with or without chemotherapy and/or brachytherapy (BT) is conventionally prescribed to patients with cervical cancer who experience local recurrence, especially those without a history of RT.

Although emerging data suggest that RT can play a critical role in treating recurrent cervical cancer, studies on the effectiveness of RT for vaginal recurrence in patients who previously underwent surgery, as well as on survival, remain limited [16, 17]. Moreover, few studies have focused on the effect of RT dose on patient survival; whether significantly higher doses would provide more effective treatment would guide future formulations of RT regimens.

In the present study, we retrospectively analyzed the data of patients with cervical cancer who experienced vaginal recurrence after hysterectomy and were treated with salvage RT at the Peking Union Medical College Hospital (PUMCH). The study's first aim was to investigate the efficacy of salvage RT in patients with cervical SCC who experienced vaginal recurrence; the second was to identify prognostic factors, including those related to RT dose.

\section{Methods}

\subsection{Patient selection}

Medical records of 177 women with cervical cancer who experienced vaginal recurrence and were treated with salvage RT at Peking Union Medical College Hospital (PUMCH) between July 2011 and November 2019 were reviewed. The following inclusion criteria were applied: (1) both initial treatment and recurrence were histologically proven SCC-subtype cervical cancer, (2) the patient had undergone hysterectomy for cervical cancer, and (3) the patient had experienced vaginal recurrence and was consequently treated with salvage RT. We excluded patients who (1) underwent hysterectomy for benign disease or did not complete surgery, (2) had a history of RT, (3) had undergone other treatments for vaginal recurrence before or after salvage RT such as tumorectomy or drugs (including chemotherapy, targeted therapy, and 
immunotherapy), and (4) had experienced recurrence in the form of distant metastasis. Ultimately, 97 patients met the inclusion criteria and were included in our study.

\subsection{Treatment}

All patients with recurrence received salvage RT as EBRT, BT, or both at PUMCH. EBRT and BT combined were delivered to 76 of the 97 participants. Fifteen patients received only EBRT, while BT alone was performed in 6 patients. Cisplatin-based concurrent chemoradiotherapy was administered to 54 patients.

All patients who received EBRT were treated with volumetric modulated arc therapy or helical tomotherapy $(n=91)$. In one patient with a relatively small tumor, EBRT was delivered only to the vaginal and paravaginal regions with a clinical target volume (CTV) of 30.0 Gy in 10 fractions, while BT was delivered at a dose of $20 \mathrm{~Gy}$ in 4 fractions. All other patients received whole pelvic EBRT (n=90). The CTVs ranged from $45.0 \mathrm{~Gy}$ to $50.4 \mathrm{~Gy}$ (mostly $50.4 \mathrm{~Gy}$ ), with $1.8 \mathrm{~Gy}$ per fraction, while the median gross tumor volume was $50.4 \mathrm{~Gy}$ (range, 45.0-80.2 Gy) boosted by EBRT. Fifteen patients received lymph node boosts with doses ranging from 43.0 to $70.7 \mathrm{~Gy}$.

Eighty-two patients received BT after vaginal recurrence either by $2 D$ conventional BT ( $n=55)$ or 3D BT ( $n=27)$. Most applicators were multichannel vaginal cylinders $(n=62)$, and a few patients adopted 3D-printed individual vaginal applicators $(n=20)$. The BT dose was usually 5 Gy (range, 3-6 Gy) per fraction, administered in 2-6 fractions.

When calculating the total dose of EBRT and BT, the biologically equivalent dose in 2 Gy fractions (EQD2) was utilized; the total EQD2 was the sum of the EQD2 values for EBRT and BT. The EQD2 dose and number of fractions for EBRT were based on the gross tumor volume data, and the minimum dose covering $90 \%$ of the CTV (CTV D90) in EQD2 was used as the representative dose of image-guided BT.

\subsection{Follow-up and statistical analysis}

The primary endpoint was overall survival (OS), while the secondary endpoints were progression-free survival (PFS) and local control (LC). OS was calculated from the date of recurrence to that of death from any cause or that of the last clinical follow-up. PFS was defined as the interval between the date of starting salvage RT and that of any recurrence, disease progression, or death. LC was calculated from the start of salvage RT to the date on which local tumor progression was detected. OS, PFS, and LC were estimated using the Kaplan-Meier method, and differences in prognosis between subgroups were compared using log-rank tests via univariate analyses. Multivariate analyses were conducted using the Cox proportional hazards regression model to identify independent prognostic factors. All statistical tests were two-sided, and statistical significance was set at a two-sided $P$-value $<0.05$. All data analyses were performed using SPSS for Windows (version 23.0 ; IBM Corp., Armonk, NY, USA).

\section{Results}

\subsection{Patient and treatment characteristics}

The patients' detailed demographic and clinical features are summarized in Table 1. Ninety-seven patients with a median age of 53 years were retrospectively reviewed. Patients were classified into 3 groups according to the recurrence pattern: endovaginal recurrence ( $n=64)$; paravaginal recurrence, in which the tumor invaded paravaginal tissues or extended from the top of the vagina toward the pelvic cavity ( $\mathrm{n}=23$ ); and invasive recurrence, in which the tumors involved surrounding organs such as the bladder, rectum, and pelvic wall ( $\mathrm{n}=10$ ). The tumor sizes of most patients were $\leq 4 \mathrm{~cm}(\mathrm{n}=82)$, whereas bulky tumors larger than $4 \mathrm{~cm}$ were observed in 15 patients. The median interval between the date of primary hysterectomy and that of recurrence was 26.4 months (range, 2.5-238.0 months). Salvage RT was performed with a median EQD2 of 69.4 Gy (range, 37.5-88.9 Gy). 
Patient and treatment characteristics.

\begin{tabular}{|c|c|c|}
\hline Characteristics & Number & Percentage \\
\hline$\leq 4 \mathrm{~cm}$ & 82 & 84.5 \\
\hline$>4 \mathrm{~cm}$ & 15 & 15.5 \\
\hline Endovaginal & 64 & 66.0 \\
\hline Paravaginal (include the top of vagina) & 23 & 23.7 \\
\hline Invasion of surrounding organs (such as bladder, rectum, pelvic wall) & 10 & 10.3 \\
\hline No & 69 & 71.1 \\
\hline \multicolumn{3}{|l|}{ RT treatment } \\
\hline $\mathrm{EBRT}+\mathrm{BT}$ & 76 & 78.3 \\
\hline EBRT & 15 & 15.5 \\
\hline BT & 6 & 6.2 \\
\hline RT dose (EQD2, Gy), median (range) & $69.4(37$ & \\
\hline $\mathrm{EBRT}+\mathrm{BT}$ & $70.0(53$ & \\
\hline \multicolumn{3}{|l|}{ BT technique } \\
\hline No & 15 & 15.5 \\
\hline \multicolumn{3}{|l|}{ Concurrent chemoradiotherapy } \\
\hline Yes & 54 & 55.7 \\
\hline No & 43 & 44.3 \\
\hline
\end{tabular}

\subsection{Clinical outcomes}

The median follow-up time was 42.5 months (range, 2.0-110.9 months). The estimated OS, PFS, and LC rates were $88 \%$, $84 \%$, and $91 \%$, respectively, at 3 years and $84 \%, 79 \%$, and 91\%, respectively, at 5 years (Fig. 1); moreover, 12 patients died. Seventeen patients experienced disease progression during the follow-up period; 4 had local recurrence, 9 had distant metastasis, and 4 experienced both.

\subsection{Factors associated with OS, PFS, and LC}

Prognostic factors including tumor size $(\leq 4 \mathrm{~cm}$ vs. $>4 \mathrm{~cm}$ ), recurrence pattern (endovaginal, paravaginal, or invasion of the surrounding organs), lymph node metastasis, RT regimen (ERBT, BT, or both), BT technique (2D or 3D), and administration of concurrent chemoradiotherapy were assessed.

Tumor size and recurrence pattern were found to be significantly associated with OS on univariate analysis, although these associations were not significant on multivariate analysis (Table 2). However, both univariate analysis and multivariate analyses results demonstrated that a 
tumor size $\leq 4 \mathrm{~cm}(P<0.05)$ and an endovaginal recurrence pattern $(P<0.05)$ were significantly associated with longer PFS (Table 2$)$. Lastly, only the recurrence pattern $(P<0.05)$ was an independent predictor of LC on univariate and multivariate analyses $(T a b l e ~ 2)$.

Table 2

Factors predictive of overall survival, progression-free survival and local control.

\begin{tabular}{|c|c|c|c|c|c|c|c|c|c|}
\hline \multirow[t]{2}{*}{ Variables } & \multirow{2}{*}{$\begin{array}{l}\text { Median } \\
\text { OS } \\
\text { (mo) }\end{array}$} & \multicolumn{2}{|l|}{$P$ value } & \multirow{2}{*}{$\begin{array}{l}\text { Median } \\
\text { PFS } \\
\text { (mo) }\end{array}$} & \multicolumn{2}{|l|}{$P$ value } & \multirow{2}{*}{$\begin{array}{l}\text { Median } \\
\text { LC } \\
\text { (mo) }\end{array}$} & \multicolumn{2}{|l|}{$P$ value } \\
\hline & & $\begin{array}{l}\text { Univariate } \\
\text { (OS) }\end{array}$ & $\begin{array}{l}\text { Multivariate } \\
\text { (OS) }\end{array}$ & & $\begin{array}{l}\text { Univariate } \\
\text { (PFS) }\end{array}$ & $\begin{array}{l}\text { Multivariate } \\
\text { (PFS) }\end{array}$ & & $\begin{array}{l}\text { Univariate } \\
\text { (LC) }\end{array}$ & $\begin{array}{l}\text { Multivariate } \\
\text { (LC) }\end{array}$ \\
\hline \multicolumn{10}{|l|}{ Tumor size } \\
\hline$\leq 4 \mathrm{~cm}$ & 42.89 & 0.002 & 0.118 & 42.43 & $<0.001$ & 0.019 & 42.43 & 0.067 & \\
\hline$>4 \mathrm{~cm}$ & 37.52 & & & 25.46 & & & 28.22 & & \\
\hline \multicolumn{10}{|c|}{ Recurrence pattern } \\
\hline Endovaginal & 45.31 & 0.013 & 0.241 & 44.78 & 0.001 & 0.041 & 44.78 & 0.011 & 0.040 \\
\hline Paravaginal & 32.49 & & 0.093 & 30.39 & & 0.012 & 30.39 & & 0.028 \\
\hline $\begin{array}{l}\text { Invasion of } \\
\text { surrounding } \\
\text { organs }\end{array}$ & 27.81 & & 0.416 & 19.75 & & 0.246 & 23.51 & & 0.020 \\
\hline \multicolumn{10}{|l|}{$\begin{array}{l}\text { Lymph nodes } \\
\text { metastasis }\end{array}$} \\
\hline Yes & 31.82 & 0.349 & & 26.23 & 0.225 & & 31.18 & 0.975 & \\
\hline No & 45.31 & & & 44.26 & & & 44.75 & & \\
\hline \multicolumn{10}{|l|}{ RT treatment } \\
\hline EBRT+BT & 40.04 & 0.144 & & 41.63 & 0.412 & & 42.43 & 0.676 & \\
\hline EBRT & 26.94 & & & 25.58 & & & 23.00 & & \\
\hline BT & 64.92 & & & 64.92 & & & 64.92 & & \\
\hline \multicolumn{10}{|l|}{ BT technique } \\
\hline $2 \mathrm{D}$ & 58.68 & 0.123 & & 58.02 & 0.347 & & 58.68 & 0.524 & \\
\hline $3 D$ & 27.00 & & & 23.98 & & & 25.50 & & \\
\hline No BT & 26.94 & & & 24.58 & & & 23.00 & & \\
\hline \multicolumn{10}{|c|}{$\begin{array}{l}\text { Concurrent } \\
\text { chemoradiotherapy }\end{array}$} \\
\hline Yes & 31.18 & 0.117 & & 25.51 & 0.151 & & 27.58 & 0.424 & \\
\hline No & 49.28 & & & 49.02 & & & 49.28 & & \\
\hline
\end{tabular}

\subsection{Effect of dose on prognosis}

Since the recurrence pattern was a significant independent predictor of both PFS and LC in our study, we stratified patients based on this factor to identify a dose cut-off. Endovaginal recurrence was associated with prolonged survival (the estimated 5-year PFS and LC rates were $90 \%$ and $97 \%$, respectively), while recurrences that were paravaginal and those that invaded surrounding organs were negative prognostic factors, with estimated 5-year PFS and LC rates of $58 \%$ and $81 \%$, respectively. Thirty-three patients with recurrences that were paravaginal or invasive of the surrounding organs were evaluated for the aforementioned prognostic factors as well as dose (EQD2 <70 Gy vs. $\geq 70 \mathrm{~Gy}$ ). On univariate analysis, a dose $\geq 70$ Gy was significantly associated with improved LC $(P=0.022 ;$ Fig. 2$)$, while none of the other factors were found to be significantly prognostic.

\subsection{Toxicity and late complications}


Twelve patients (12.4\%) experienced grade $\geq 2$ late complications. Although the rates of grade $\geq 2$ late complications were similar between patients treated with combination EBRT and BT and those who only underwent BT (13.2\% and $13.3 \%$, respectively), one patient in the EBRTonly group experienced a grade 5 late complication (a rectovaginal fistula), whereas no such complications were observed in patients who received $\mathrm{BT}$ alone (Table 3 ). Among the 33 patients with recurrent tumors that were paravaginal or invasive of surrounding organs, there was no obvious difference in the rates of grade $\geq 2$ late complications between those who received EQD2 $\geq 70$ Gy and those who received $<70$ Gy (Table S1).

Table 3

Late complications in patients who underwent various radiotherapy regimens.

\begin{tabular}{|c|c|c|c|}
\hline Late complication & EBRT+BT $(n=76)$ & EBRT $(n=15)$ & BT $(n=6)$ \\
\hline \multicolumn{4}{|c|}{ Lower gastrointestinal toxicity } \\
\hline Grade 0 & $77.6 \%(59 / 76)$ & $66.7 \%(10 / 15)$ & $100 \%(6 / 6)$ \\
\hline Grade 1 & $13.2 \%(10 / 76)$ & $20 \%(3 / 15)$ & 0 \\
\hline Grade 2 & $7.9 \%(6 / 76)$ & $6.7 \%(1 / 15)$ & 0 \\
\hline Grade 3 & $1.3 \%(1 / 76)$ & 0 & 0 \\
\hline Grade 4 & 0 & 0 & 0 \\
\hline Grade 5 & 0 & $6.7 \%(1 / 15)$ & 0 \\
\hline \multicolumn{4}{|l|}{ Urinary tract toxicity } \\
\hline Grade 0 & $81.6 \%(62 / 76)$ & $93.3 \%(14 / 15)$ & $100 \%(6 / 6)$ \\
\hline Grade 1 & $13.2 \%(10 / 76)$ & $6.7 \%(1 / 15)$ & 0 \\
\hline Grade 2 & $5.3 \%(4 / 76)$ & 0 & 0 \\
\hline Grade 3 & 0 & 0 & 0 \\
\hline \multicolumn{4}{|c|}{ Hematological toxicity } \\
\hline Grade 0 & $86.8 \%(66 / 76)$ & $86.7(13 / 15)$ & $100 \%(6 / 6)$ \\
\hline Grade 1 & $9.2 \%(7 / 76)$ & $13.3 \%(2 / 15)$ & 0 \\
\hline Grade 2 & $1.3 \%(1 / 76)$ & 0 & 0 \\
\hline Grade 3 & $2.6 \%(2 / 76)$ & 0 & 0 \\
\hline
\end{tabular}

\section{Discussion}

RT has been shown to be an effective treatment for patients with cervical cancer who experience locoregional recurrence after surgery. In a study by Kim et al., the 5-year local failure-free survival and OS rates of patients with cervical cancer who underwent RT after experiencing post-surgical recurrence were $63.9 \%$ and $66 \%$, respectively [18]. In another study, Kim et al. found that the 5-year PFS and OS rates after RT were $62.7 \%$ and $60.1 \%$, respectively [13]. These studies showed far better survival rates than did those performed in the previous decade in which the 5-year OS rates were below 50\% $[16,17]$. Our study focused on RT-naïve patients with cervical SCC; these individuals achieved excellent estimated 5-year OS, PFS, and LC rates of $84 \%, 79 \%$, and $91 \%$, respectively. These superior clinical outcomes can be partially attributed to the pathological tumor type, higher RT dose, and lack of previous RT. The 5-year local failure-free survival and PFS rates for reirradiated patients in Kim et al.'s study were only $47.1 \%$ and $33.2 \%$, respectively, indicating that having previously received RT had a negative effect on treatment, likely owing to dose limitations and radiosensitivity [18].

Although surgeries such as pelvic exenteration are also treatment options for patients with cervical cancer who experience central pelvic relapse without pelvic wall involvement or extrapelvic spread, RT has its own advantages (most notably fewer complications). Pelvic exenteration is usually considered the only curative therapeutic approach, but it is associated with high surgery-related morbidity and mortality rates [19]; the survival rate is less than $50 \%$, while mortality is nearly $5 \%[12,20]$. Other surgical methods such as laterally extended pelvic/endopelvic resection carry certain risks of injury to the nerve, bladder, or rectum, leading to impaired urination and defecation. Moreover, a shortened vaginal length after surgery greatly reduces the quality of life as well as the female sexual function index [21]. The curative effect of surgery is also limited in patients with human papillomavirus-associated cervical cancer, as only the upper segments of the vagina with 
visible tumor can be resected while the lower, virus-infected segments may continue to undergo oncogenic transformation, resulting in rerecurrence. As such, RT can address these limitations of surgery.

We found that the factors that significantly influenced the effectiveness of RT for patients with recurrence were tumor size and recurrence pattern. Previous studies have shown that an initial tumor diameter $\leq 4 \mathrm{~cm}$ was a predictor of favorable LC in patients with cervical cancer who experienced advanced vaginal recurrence [22]. In Zolciak-Siwinska et al.'s study, a recurrent tumor diameter $>3 \mathrm{~cm}$ was a significant prognostic indicator as it negatively influenced OS, DFS, and LC in patients with recurrences in the cervix or vagina who underwent re-irradiation [23]. In our study, endovaginal recurrence was a significant predictor of more favorable OS, PFS, and LC. The treatment dose for tumors that recur extravaginally is dependent on the surrounding organs such as the bladder, rectum, and intestine; therefore, treatment is more difficult in such patients than it is in subjects who only require radical RT.

Since recurrent tumors that are paravaginal or invasive of surrounding organs are associated with a relatively poor prognosis, it was an important objective of our study to explore treatment that could improve survival in these patients. Through stratified analysis, we discovered that the RT dose significantly affected LC in patients with extravaginal recurrence; this was a most novel finding of this study. Few investigations have elucidated the relationship between RT dose and survival; hence, ours was the first to show that a higher EQD2 ( $\geq 70$ Gy) was significantly associated with prolonged LC. Presently, EBRT and BT combined constitute the first radical concurrent chemoradiotherapy choice for patients with locally advanced cervical cancer; however, there is no consensus on the optimal mode of RT for central recurrent cervical cancer, or on whether EBRT and BT should be administered [11, 13, 15, 18, 24]. Compared to 3-dimensional conformal radiation therapy, intensity-modulated RT reduces the doses to (and irradiation volumes of) the small intestine, rectum, and bone marrow [25, 26], reducing the risk of acute and chronic gastrointestinal and urinary toxicity $[27,28]$. However, intensity-modulated RT is not a substitute for BT [29]. The latter allows for a higher dose of radiation to the tumor while minimizing toxicity to adjacent tissues. Moreover, our study found that combination EBRT and BT was safer than administering EBRT while increasing the irradiation dose. Moreover, the development of computed tomography- or magnetic resonance imaging-guided 3D intracavitary/interstitial BT and the application of new techniques such as 3D printing are expected to further optimize the dose to the tumor region while reducing toxicities and complications [30-34].

Notably, higher irradiation doses have not been definitively shown to lead to improved OS or PFS, indicating that RT has limited systemic therapeutic benefits despite excellent LC. Chemotherapy is often recommended for patients with extrapelvic metastases or recurrent disease who are not candidates for RT or exenterative surgery. Another retrospective analysis performed at our institution found that the PFS of patients with intrapelvic cervical cancer recurrence who were treated with RT alone was superior to that of counterparts who underwent a combination of RT and chemotherapy $(P=0.005)$ [19]. Multivariate analysis of data from that study did not find that concurrent chemotherapy was superior to RT alone. However, it has been shown that the tumor volume before BT is an important prognostic factor in patients with advanced cervical cancer and that their prognosis is more favorable when the high-risk CTV is $\leq 30 \mathrm{cc}[35,36]$. With the increase in volume, a higher RT dose is required to achieve an LC rate over $90 \%$ [35, 36]. Platinum-based concurrent chemotherapy could increase sensitivity and reduce distant metastasis [37] and might be able to shrink the tumor volume faster and achieve potential survival benefits, especially in patients with extravaginal recurrence.

The limitations of this study include its retrospective, non-randomized design and inconsistent management of treatment owing to the long duration of the study. Second, potential bias may have been introduced during patient selection for the initial surgery and treatment of recurrence. Third, there have been continuous advances in RT technology over the time span of the study, and the type of RT administered (i.e., EBRT with or without BT or 2D-BT versus 3D-BT) as well as the dose distribution and fractionation of EBRT and BT were designed according to both the tumor characteristics and RT instrumentation available at the time. Thus, it was difficult to analyze whether a specific type of RT technology or dose fractionation influenced survival; as such, additional, prospective multicenter studies are warranted.

\section{Conclusions}

In conclusion, we found salvage RT to be an effective treatment for vaginal recurrences in patients with pathologically confirmed cervical SCC who had previously undergone surgery. The recurrence pattern and tumor size were significant prognostic factors. For patients with extravaginal recurrence, a dose of $\geq 70 \mathrm{~Gy}$ is recommended; moreover, combined EBRT and BT might be a more suitable option.

\section{Abbreviations}

\section{SCC}

squamous cell carcinoma

RT

radiotherapy

EBRT 
external beam radiotherapy

BT

brachytherapy

CTV

clinical target volume

EQD2

equivalent dose of 2 Gy per fraction

OS

overall survival

PFS

progression-free survival

LC

local control.

\section{Declarations}

\section{Ethics approval and consent to participate}

The study was conducted according to the guidelines of the Declaration of Helsinki, and approved by the Institutional Ethics Committee of Peking Union Medical College Hospital (HS2020123; Jan 6th, 2021).

\section{Consent for publication}

Informed consent was obtained from all subjects involved in the study. Written informed consent has been obtained from the patients to publish this paper.

\section{Availability of data and materials}

All data used in the study are already provided in the tables, figures, and online supplementary materials.

\section{Competing interests}

The authors have no potential conflicts of interest to report.

\section{Funding}

This work was funded by the Education Reform Program (No. 2020zlgc0124) of Peking Union Medical College and the National Key Research and Development Plan, Ministry of Science and Technology of the People's Republic of China (No. 2016YFC0105207). There was no financing from public funds or third parties.

\section{Authors' contributions}

Junfang Yan and Ziye Zheng collected, analyzed, and interpreted the data and wrote the manuscript. Ke Hu and Xiaorong Hou reviewed and edited the manuscript. Lihua Yu assisted in patient follow-up. Fuquan Zhang designed the study, interpreted the data, critically reviewed the manuscript, and supervised the study. Fuquan Zhang has full access to all the data in the study and the final responsibility for the decision to submit for publication. All authors read and approved the final manuscript.

\section{Acknowledgements}

We appreciate the support of PUMCH and the effort of every medical stuff who have treated these patients.

\section{References}

1. Bray F, Ferlay J, Soerjomataram I, Siegel RL, Torre LA, Jemal A. Global cancer statistics 2018: GLOBOCAN estimates of incidence and mortality worldwide for 36 cancers in 185 countries. CA Cancer J Clin. 2018;68(6):394-424.

2. Li D, Xu X, Yan D, Yuan S, Ni J, Lou H. Prognostic factors affecting survival and recurrence in patients with early cervical squamous cell cancer following radical hysterectomy. J Int Med Res. 2020;48(4):300060519889741.

3. Small W Jr, Bacon MA, Bajaj A, Chuang LT, Fisher BJ, Harkenrider MM, et al. Cervical cancer: A global health crisis. Cancer. 2017;123(13):2404-12. 
4. Davy ML, Dodd TJ, Luke CG, Roder DM. Cervical cancer: effect of glandular cell type on prognosis, treatment, and survival. Obstet Gynecol. 2003;101(1):38-45.

5. Nakanishi T, Ishikawa H, Suzuki Y, Inoue T, Nakamura S, Kuzuya K. A comparison of prognoses of pathologic stage lb adenocarcinoma and squamous cell carcinoma of the uterine cervix. Gynecol Oncol. 2000;79(2):289-93.

6. Lee YY, Choi CH, Kim TJ, Lee JW, Kim BG, Lee JH, et al. A comparison of pure adenocarcinoma and squamous cell carcinoma of the cervix after radical hysterectomy in stage IB-IIA. Gynecol Oncol. 2011;120(3):439-43.

7. Galic V, Herzog TJ, Lewin SN, Neugut Al, Burke WM, Lu YS, et al. Prognostic significance of adenocarcinoma histology in women with cervical cancer. Gynecol Oncol. 2012;125(2):287-91.

8. Ramirez PT, Frumovitz M, Pareja R, Lopez A, Vieira M, Ribeiro R, et al. Minimally Invasive versus Abdominal Radical Hysterectomy for Cervical Cancer. N Engl J Med. 2018;379(20):1895-904.

9. Thomas GM, Dembo AJ, Myhr T, Black B, Pringle JF, Rawlings G. Long-term results of concurrent radiation and chemotherapy for carcinoma of the cervix recurrent after surgery. Int J Gynecol Cancer. 1993;3(4):193-8.

10. Mahdi H, Rose PG, Elshaikh MA, Munkarah A, Isrow D, Singh S, et al. Adjuvant vaginal brachytherapy decreases the risk of vaginal recurrence in patients with stage I non-invasive uterine papillary serous carcinoma. A multi-institutional study. Gynecol Oncol. 2015;136(3):529-33.

11. Cohen PA, Jhingran A, Oaknin A, Denny L. Cervical cancer. The Lancet. 2019;393(10167):169-82.

12. Benn T, Brooks RA, Zhang Q, Powell MA, Thaker PH, Mutch DG, et al. Pelvic exenteration in gynecologic oncology: a single institution study over 20 years. Gynecol Oncol. 2011;122(1):14-8.

13. Kim SW, Chun M, Ryu HS, Chang SJ, Kong TW, Lee EJ, et al. Salvage radiotherapy with or without concurrent chemotherapy for pelvic recurrence after hysterectomy alone for early-stage uterine cervical cancer. Strahlenther Onkol. 2017;193(7):534-42.

14. Kobayashi K, Murakami N, Takahashi K, Inaba K, Hamamoto R, Itami J. Local Radiotherapy or Chemotherapy for Oligo-recurrent Cervical Cancer in Patients With Prior Pelvic Irradiation. In vivo (Athens. Greece). 2019;33(5):1659-65.

15. Abu-Rustum NR, Yashar CM, Bean S, Bradley K, Campos SM, Chon HS, et al. NCCN Guidelines Insights: Cervical Cancer, Version 1.2020. J Natl Compr Canc Netw. 2020;18(6):660-6.

16. Haasbeek CJ, Uitterhoeve AL, van der Velden J, Gonzalez DG, Stalpers LJ. Long-term results of salvage radiotherapy for the treatment of recurrent cervical carcinoma after prior surgery. Radiother Oncol. 2008;89(2):197-204.

17. Jain P, Hunter RD, Livsey JE, Coyle C, Swindell R, Davidson SE. Salvaging locoregional recurrence with radiotherapy after surgery in early cervical cancer. Clin Oncol (R Coll Radiol). 2007;19(10):763-8.

18. Kim HJ, Chang JS, Koom WS, Lee KC, Kim GE, Kim YB. Radiotherapy is a safe and effective salvage treatment for recurrent cervical cancer. Gynecol Oncol. 2018;151(2):208-14.

19. Chao X, Song X, Wu H, You Y, Wu M, Li L. Selection of Treatment Regimens for Recurrent Cervical Cancer. Front Oncol. 2021;11:618485.

20. Morley GW, Hopkins MP, Lindenauer SM, Roberts JA. Pelvic exenteration, University of Michigan: 100 patients at 5 years. Obstet Gynecol. 1989;74(6):934-43.

21. Zhang Y, Sun S, Ding J, Hua K. The Effect of Different Surgical Methods on Female and Male Sexual Activity and Marital Quality in Patients With Early-Stage Cervical Cancer. Sex Med. 2020;8(2):307-14.

22. Weitmann HD, Knocke TH, WaldhausI C, Potter R. Ultrasound-guided interstitial brachytherapy in the treatment of advanced vaginal recurrences from cervical and endometrial carcinoma. Strahlenther Onkol. 2006;182(2):86-95.

23. Zolciak-Siwinska A, Bijok M, Jonska-Gmyrek J, Kawczynska M, Kepka L, Bujko K, et al. HDR brachytherapy for the reirradiation of cervical and vaginal cancer: analysis of efficacy and dosage delivered to organs at risk. Gynecol Oncol. 2014;132(1):93-7.

24. Hille A, Weiss E, Hess CF. Therapeutic outcome and prognostic factors in the radiotherapy of recurrences of cervical carcinoma following surgery. Strahlenther Onkol. 2003;179(11):742-7.

25. Shih KK, Hajj C, Kollmeier M, Frey MK, Sonoda Y, Abu-Rustum NR, et al. Impact of postoperative intensity-modulated radiation therapy (IMRT) on the rate of bowel obstruction in gynecologic malignancy. Gynecol Oncol. 2016;143(1):18-21.

26. Mell LK, Sirak I, Wei L, Tarnawski R, Mahantshetty U, Yashar CM, et al. Bone Marrow-sparing Intensity Modulated Radiation Therapy With Concurrent Cisplatin For Stage IB-IVA Cervical Cancer: An International Multicenter Phase II Clinical Trial (INTERTECC-2). Int J Radiat Oncol Biol Phys. 2017;97(3):536-45.

27. Klopp AH, Yeung AR, Deshmukh S, Gil KM, Wenzel L, Westin SN, et al. Patient-Reported Toxicity During Pelvic Intensity-Modulated Radiation Therapy: NRG Oncology-RTOG 1203. J Clin Oncol. 2018;36(24):2538-44.

28. Yeung AR, Pugh SL, Klopp AH, Gil KM, Wenzel L, Westin SN, et al. Improvement in Patient-Reported Outcomes With Intensity-Modulated Radiotherapy (RT) Compared With Standard RT: A Report From the NRG Oncology RTOG 1203 Study. J Clin Oncol. 2020;38(15):1685-92. 
29. Assenholt MS, Petersen JB, Nielsen SK, Lindegaard JC, Tanderup K. A dose planning study on applicator guided stereotactic IMRT boost in combination with 3D MRI based brachytherapy in locally advanced cervical cancer. Acta Oncol. 2008;47(7):1337-43.

30. Kim YJ, Kang HC, Kim YS. Impact of intracavitary brachytherapy technique (2D versus 3D) on outcomes of cervical cancer: a systematic review and meta-analysis. Strahlenther Onkol. 2020;196(11):973-82.

31. Jastaniyah N, Yoshida K, Tanderup K, Lindegaard JC, Sturdza A, Kirisits C, et al. A volumetric analysis of GTVD and CTVHR as defined by the GEC ESTRO recommendations in FIGO stage IIB and IIIB cervical cancer patients treated with IGABT in a prospective multicentric trial (EMBRACE). Radiother Oncol. 2016;120(3):404-11.

32. Sturdza A, Potter R, Fokdal LU, Haie-Meder C, Tan LT, Mazeron R, et al. Image guided brachytherapy in locally advanced cervical cancer: Improved pelvic control and survival in RetroEMBRACE, a multicenter cohort study. Radiother Oncol. 2016;120(3):428-33.

33. Fokdal L, Sturdza A, Mazeron R, Haie-Meder C, Tan LT, Gillham C, et al. Image guided adaptive brachytherapy with combined intracavitary and interstitial technique improves the therapeutic ratio in locally advanced cervical cancer: Analysis from the retroEMBRACE study. Radiother Oncol. 2016;120(3):434-40.

34. Liu Y, Jiang P, Zhang H, Wang J. Safety and efficacy of 3D-printed templates assisted CT-guided radioactive iodine-125 seed implantation for the treatment of recurrent cervical carcinoma after external beam radiotherapy. J Gynecol Oncol. 2021;32(2):e15.

35. Mazeron R, Castelnau-Marchand P, Dumas I, del Campo ER, Kom LK, Martinetti F, et al. Impact of treatment time and dose escalation on local control in locally advanced cervical cancer treated by chemoradiation and image-guided pulsed-dose rate adaptive brachytherapy. Radiother Oncol. 2015;114(2):257-63.

36. Tanderup K, Fokdal LU, Sturdza A, Haie-Meder C, Mazeron R, van Limbergen E, et al. Effect of tumor dose, volume and overall treatment time on local control after radiochemotherapy including MRI guided brachytherapy of locally advanced cervical cancer. Radiother Oncol. 2016;120(3):441-6.

37. Rose PG. Chemoradiotherapy for cervical cancer. Eur J Cancer. 2002;38(2):270-8.

\section{Figures}

(A)

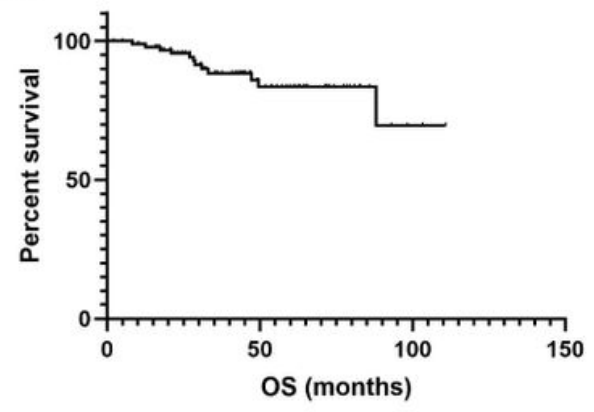

(B)

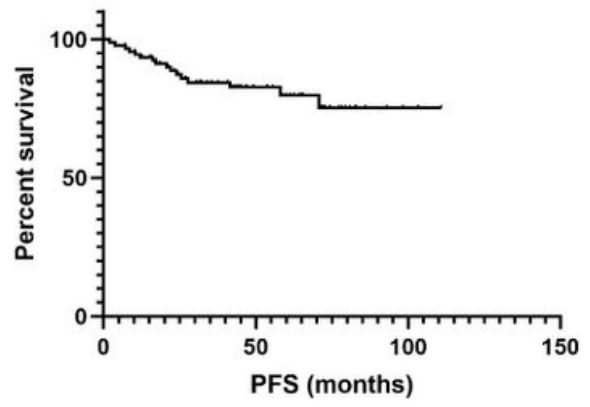

(C)

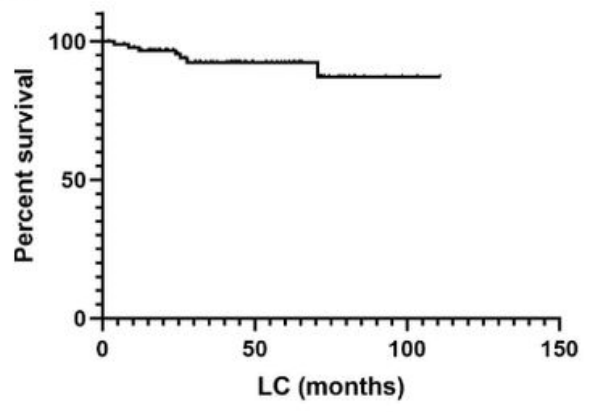

\section{Figure 1}

Kaplan-Meier curves showing (A) overall survival (OS), (B) progression-free survival (PFS), and (C) local control (LC) after salvage radiotherapy following recurrence. 


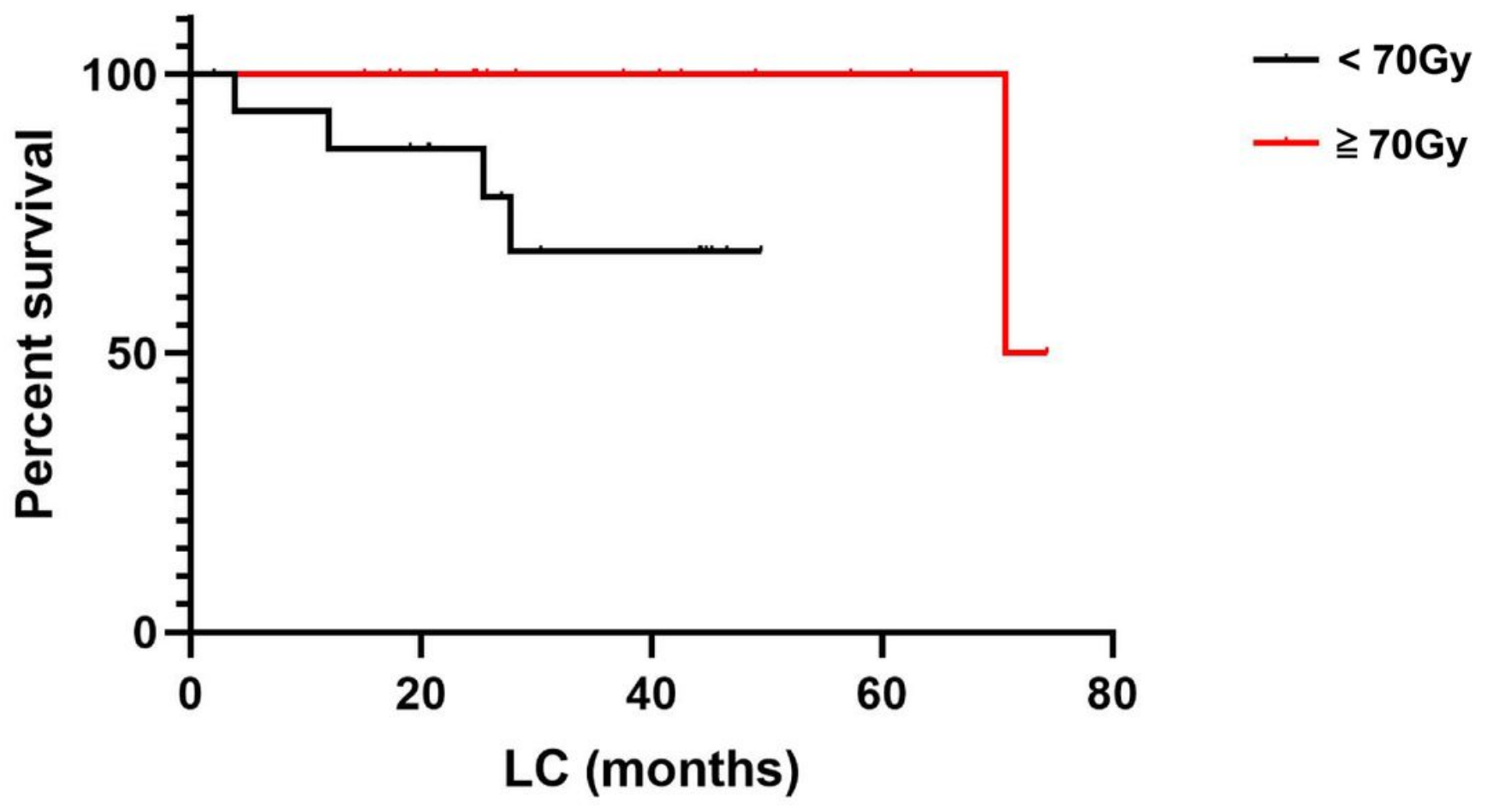

Figure 2

The local control rates of patients with paravaginal recurrence or recurrence invading surrounding organs according to equivalent dose of 2 Gy per fraction $<70$ Gy vs. $\geq 70$ Gy.

\section{Supplementary Files}

This is a list of supplementary files associated with this preprint. Click to download.

- Tables1.docx 